Supporting Information

\title{
Terminal Structure of Triethylene Glycol-Tethered Chains on $\beta$-Cyclodextrin-Threaded Polyrotaxanes Dominates Temperature-Responsivity and Biointeractions
}

\author{
Moe Ohashi, Atsushi Tamura*, Nobuhiko Yui
}

\begin{abstract}
Department of Organic Biomaterials, Institute of Biomaterials and Bioengineering, Tokyo Medical and Dental University (TMDU), 2-3-10 Kanda-Surugadai, Chiyoda, Tokyo 101-0062, Japan.

*Corresponding author: Atsushi Tamura (E-mail: tamura.org@tmd.ac.jp)
\end{abstract}

S1. Instrumentations. Size exclusion chromatography (SEC) was carried out on a Prominence-i LC2030 Plus (Shimadzu, Kyoto, Japan) equipped with an RID-20A refractive index (RI) detector and a combination of TSK gel $\alpha-4000$ and $\alpha-2500$ columns (300 mm length, $7.8 \mathrm{~mm}$ internal diameter; Tosoh, Tokyo, Japan). The system was eluted with dimethyl sulfoxide (DMSO) containing $10 \mathrm{mM} \mathrm{LiBr}$, at a flow rate of $0.35 \mathrm{~mL} / \mathrm{min}$ at $60{ }^{\circ} \mathrm{C}$. The molecular weight distribution $\left(M_{\mathrm{w}} / M_{\mathrm{n}}\right)$ was calculated using a calibration curve of standard poly(ethylene glycol) (Agilent Technologies, Wilmington, DE, USA).

Fourier-transform infrared (FT-IR) spectra were recorded using a Spectrum 100 FTIR spectrometer (Perkin Elmer, Wellesley, MA, USA) equipped with an HgCdTe (MCT) detector. The sample powders were ground with $\mathrm{KBr}$ for preparing pellets for transmission measurements.

Two-dimensional nuclear Overhauser effect spectroscopy (2D NOESY) was performed on a Bruker Advance III $400 \mathrm{MHz}$ spectrometer (Bruker BioSpin, Rheinstetten, Germany) in deuterated phosphate buffer saline (PBS; $10 \mathrm{mM} \mathrm{Na} 2 \mathrm{DPO}_{4}-\mathrm{NaD}_{2} \mathrm{PO}_{4}, 150 \mathrm{mM} \mathrm{NaCl}, \mathrm{pD} 7.0$ ) at a concentration of 10 $\mathrm{mg} / \mathrm{mL}$ at $25^{\circ} \mathrm{C}$.

S2. Dynamic Light Scattering (DLS). The number-average diameter of the TEG-PRXs were determined on a Zetasizer Nano ZS (Malvern Instruments, Malvern, UK) equipped with a $4 \mathrm{~mW} \mathrm{He}-$ Ne laser $(633 \mathrm{~nm})$. The measurements were performed at a detection angle of $173^{\circ}$ at the temperature range of $20-70{ }^{\circ} \mathrm{C}$. The TEG-PRXs were dissolved in PBS at a concentration of $10 \mathrm{mg} / \mathrm{mL}$ for DLS measurements. 
Table S1. Characterization of Cy5.5-labeled TEG-PRXs.

\begin{tabular}{|c|c|c|c|c|c|}
\hline Sample code & $\begin{array}{c}\text { Terminal group } \\
\text { of TEG-tethered } \\
\text { chains }\end{array}$ & $\begin{array}{l}\text { Number of } \\
\text { threading } \\
\beta-\mathrm{CDs}\end{array}$ & $\begin{array}{l}\text { Number of TEG- } \\
\text { tethered chains on } \\
\text { PRX (per } \beta-C D)^{a}\end{array}$ & $\begin{array}{c}\text { Number of } \\
\text { Cy5.5 on PRX }\end{array}$ & $M_{\mathrm{n}}^{\mathrm{c}}$ \\
\hline Cy5.5-HPRX & Hydroxy & 12.3 & $49.2(4.0)$ & 0.63 & 29,400 \\
\hline Cy5.5-MPRX & Methoxy & 12.3 & $55.4(4.5)$ & 0.35 & 32,000 \\
\hline Cy5.5-EPRX & Ethoxy & 12.3 & $71.3(5.8)$ & 0.36 & 34,800 \\
\hline
\end{tabular}



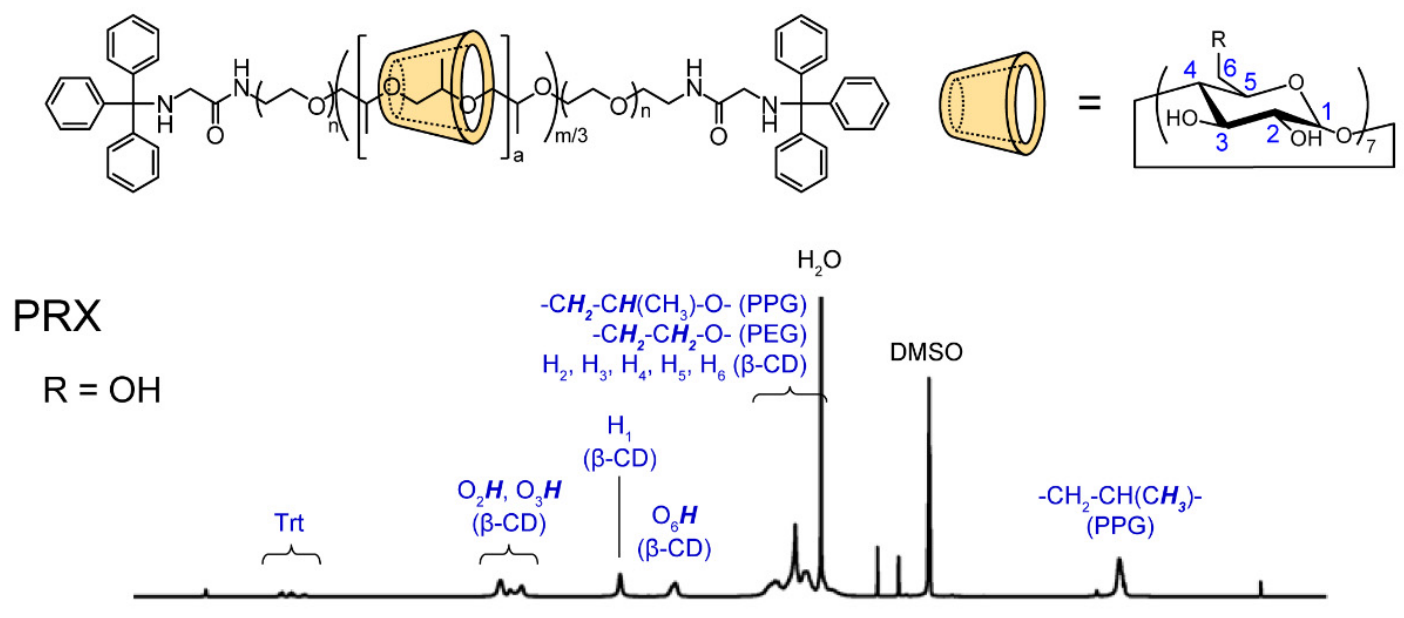

\section{HPRX}

$R=m_{O} \overbrace{H}^{O_{N}} \overbrace{\mathbf{b}}^{\mathbf{a}} \overbrace{\mathbf{b}}^{\mathbf{b}} \overbrace{\mathbf{b}}^{\mathbf{b}} \mathrm{OH}$

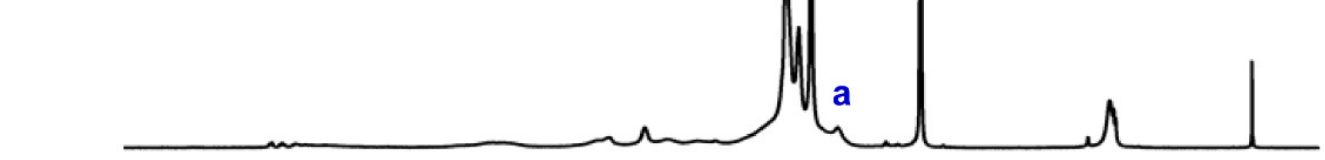

MPRX

$R=m_{0} \overbrace{H}^{\|_{N} \overbrace{d}^{c}} \overbrace{d}^{d} \overbrace{d}^{d} \overbrace{d}$

d

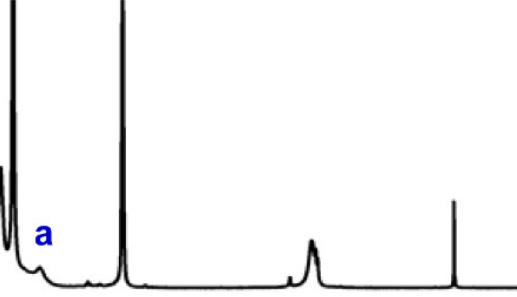




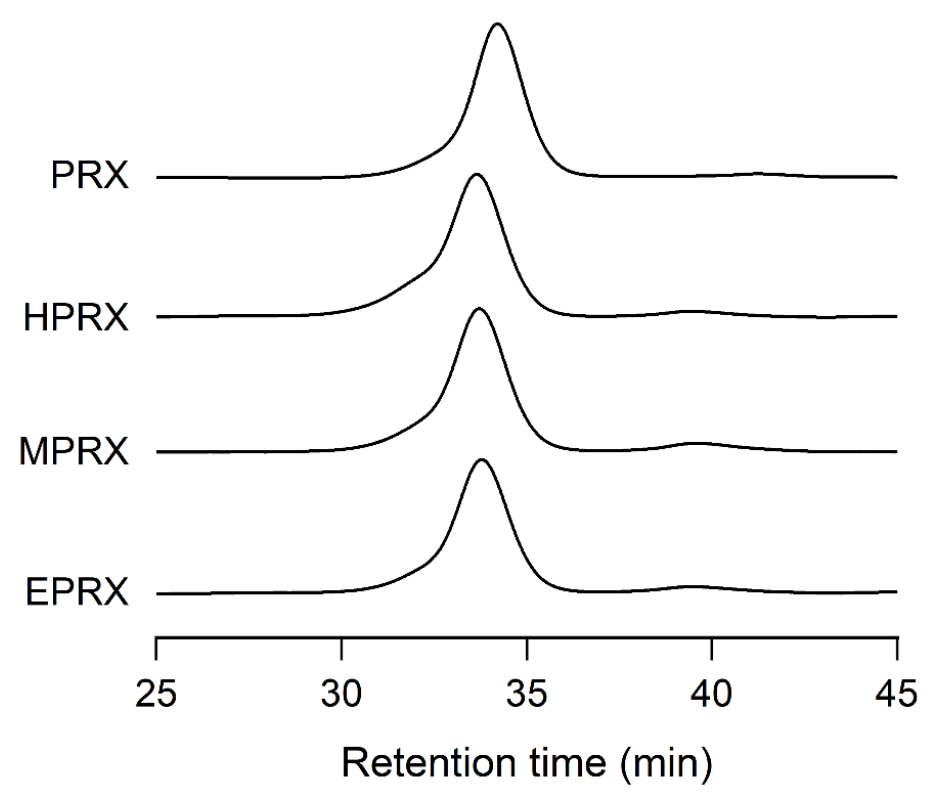

Figure S2. SEC charts of unmodified PRX, HPRX, MPRX, and EPRX in DMSO containing $10 \mathrm{mM}$ LiBr.

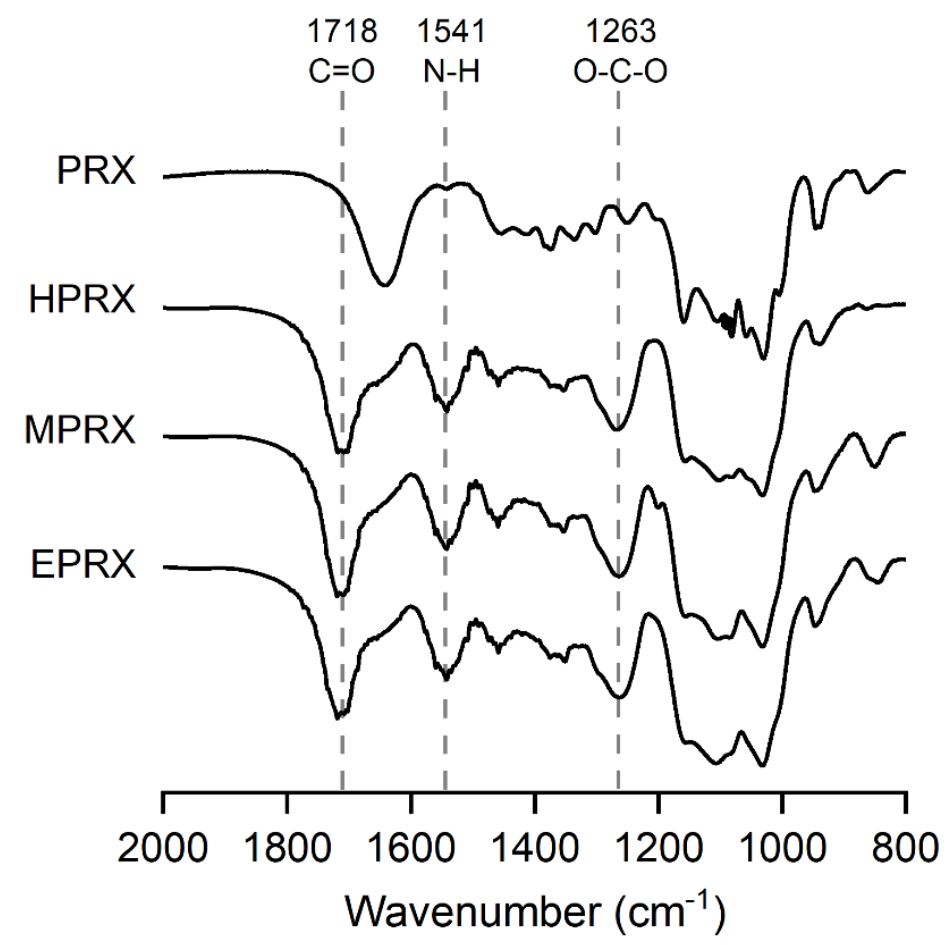

Figure S3. FT-IR spectra of unmodified PRX, HPRX, MPRX, and EPRX. 


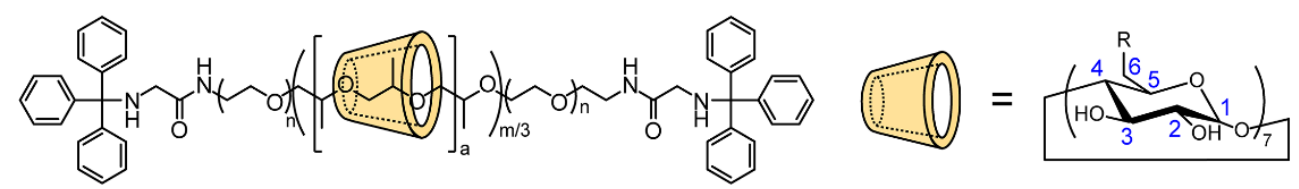

HPRX

MPRX
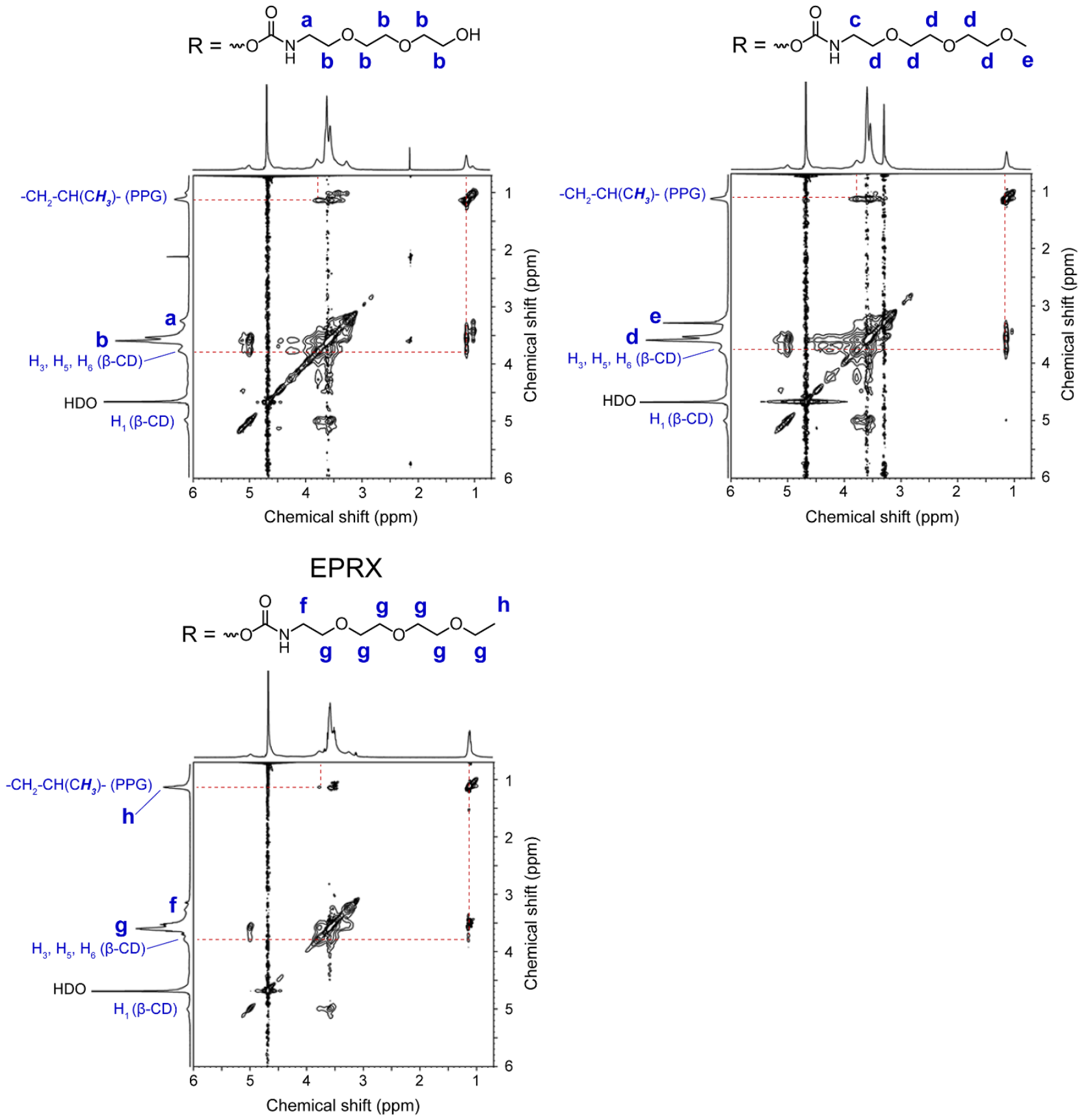

Figure S4. 2D NOESY spectra of HPRX, MPRX, and EPRX in deuterated PBS (10 mM Na2 $\mathrm{DPO}_{4}-$ $\mathrm{NaD}_{2} \mathrm{PO}_{4}, 150 \mathrm{mM} \mathrm{NaCl}, \mathrm{pD} 7.0$ ) at $25^{\circ} \mathrm{C}$. The concentration of each TEG-PRX was $10 \mathrm{mg} / \mathrm{mL}$. 


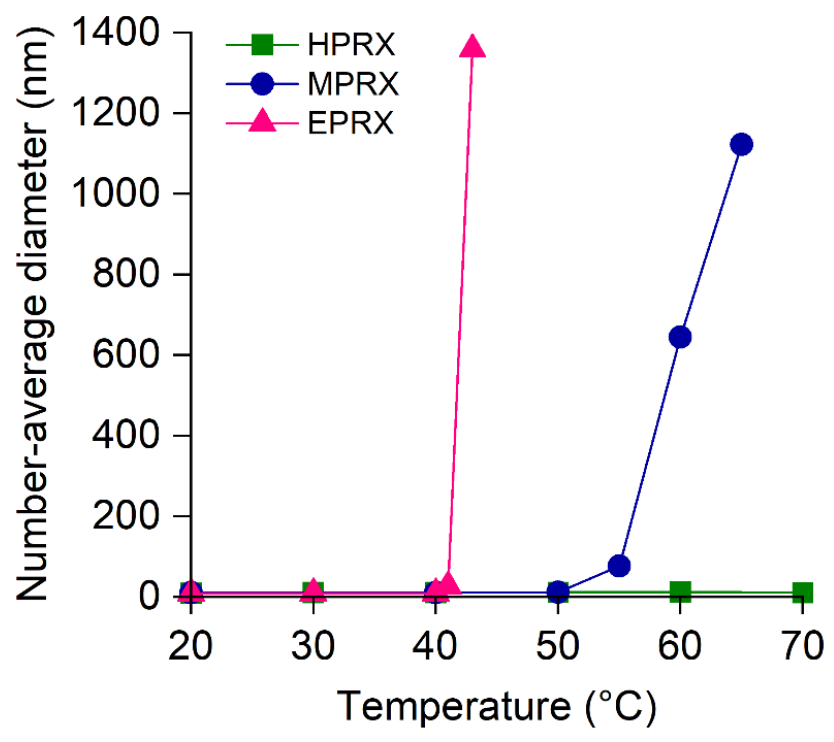

Figure S5. Temperature-dependent changes in the number-average diameter of HPRX (squares), MPRX (circles), and EPRX (triangles) in PBS.
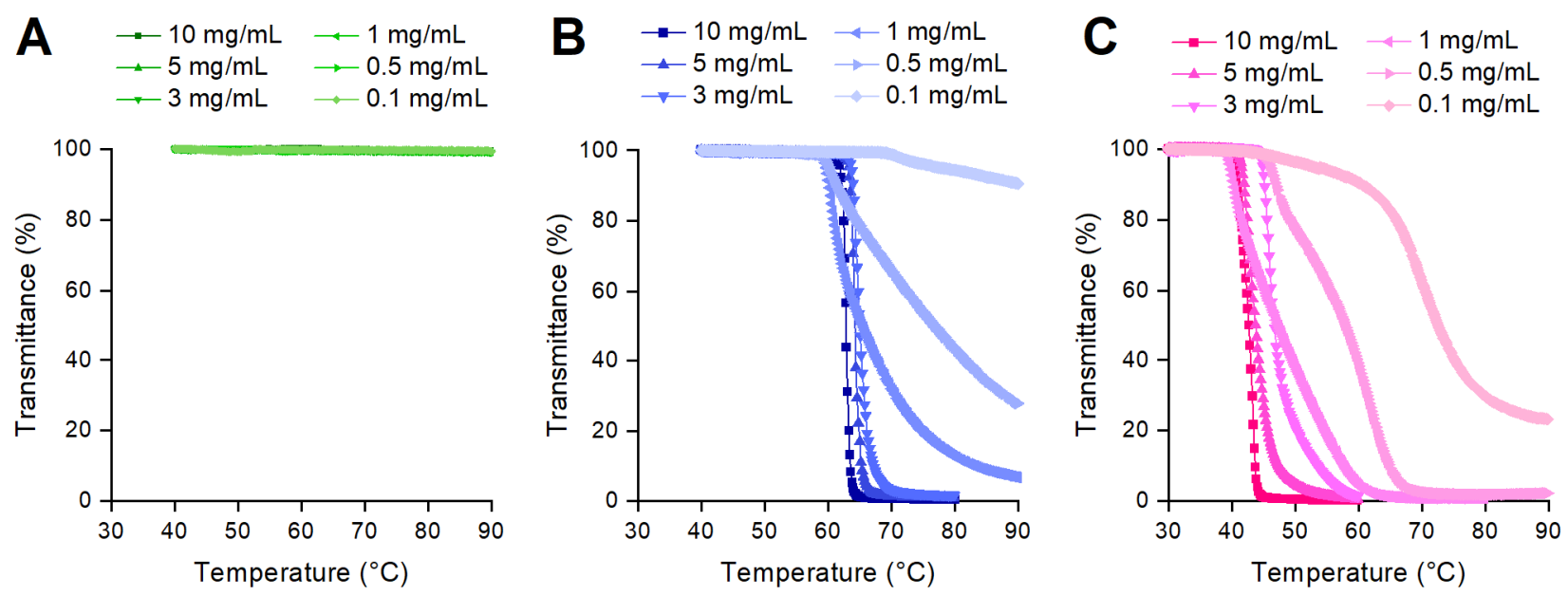

Figure S6. Temperature-dependent transmittance changes of (A) HPRX, (B) MPRX, and (C) EPRX in PBS at various concentrations $(0.1-10 \mathrm{mg} / \mathrm{mL})$. (D) Relationships between $T_{\mathrm{CP}}$ values and the concentration of MPRX (circles) and EPRX (triangles). 

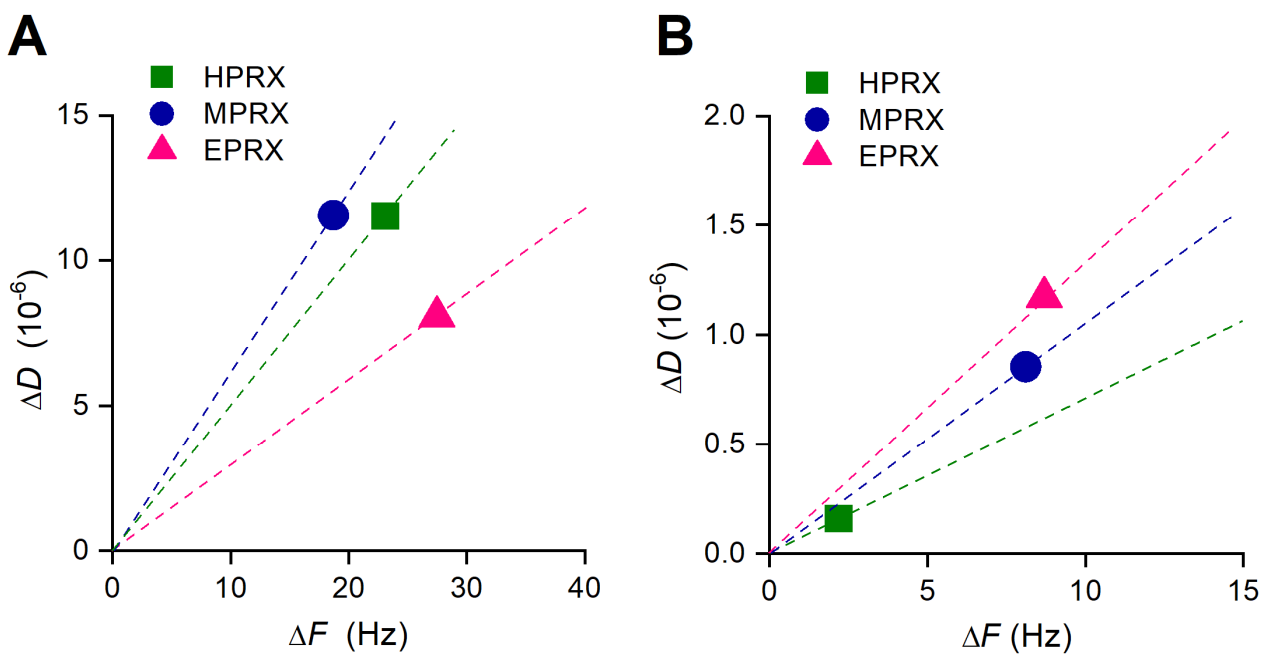

Figure S7. Relationships between $\Delta D$ and $\Delta F$ on (A) lipid-coated $\mathrm{SiO}_{2}$ surfaces and (B) BSAimmobilized gold surfaces treated with HPRX (squares), MPRX (circles), and EPRX (triangles).

A

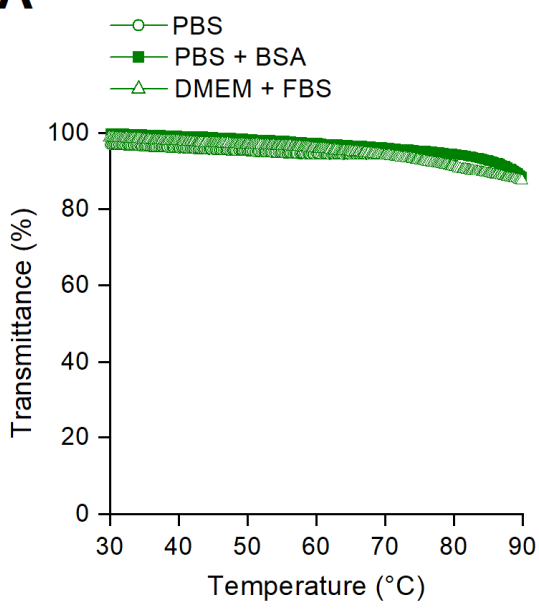

B

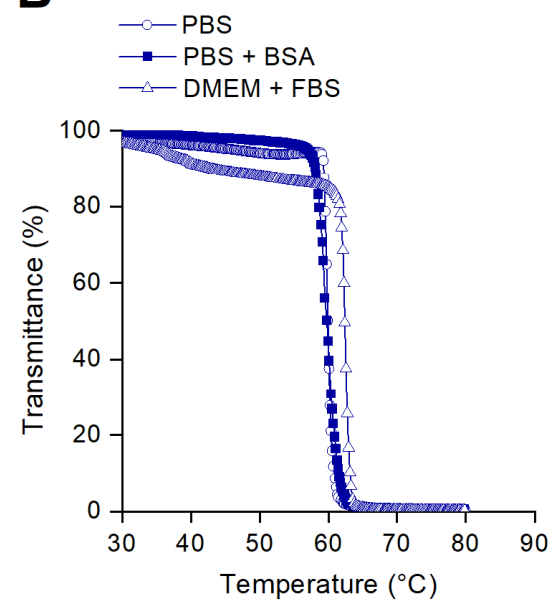

C

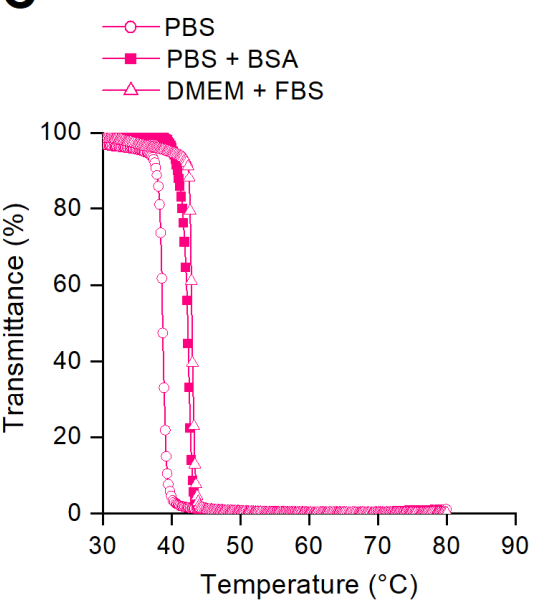

Figure S8. Temperature-dependent transmittance changes of (A) HPRX, (B) MPRX, and (C) EPRX in PBS (open circles), PBS containing $10 \mathrm{mg} / \mathrm{mL}$ BSA (closed squares), and DMEM containing 10\% FBS (open triangles). 


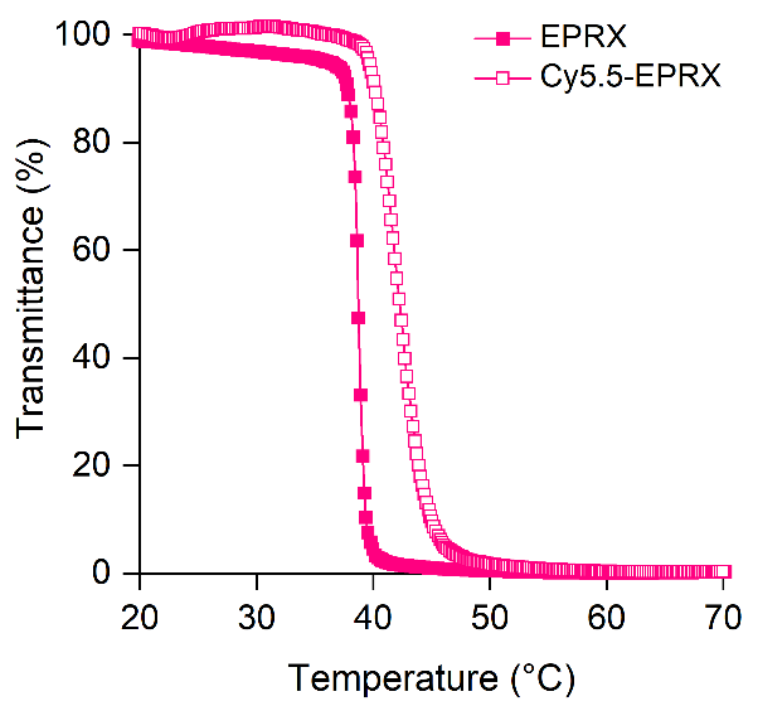

Figure S9. Temperature-dependent transmittance changes of EPRX (closed squares) and Cy5.5-EPRX (open squares) in PBS. 